noch Spuren von Harzsäuren zu erhalten. Eine kleinere Menge der rohen Resene losten wir in kaltem Alkohol und fällten wiederholt mit $\mathrm{HCl}$-haltigem Wasser. Den gut ausgewaschenen Niederschlag trockneten wir ohne Anwendung ron Wäme und verwandten die Substanzen erst dann zur Elementaranalyse, nachdem ihr Schmelzpunkt auch nicht die geringste Aenderung mehr zeigte. Die ủber Schwefelsăure getrockneten Substanzen ergaben dann die aus der Tabelle ersichtlichen Resultate. Wir erhielten so ziemlich ubereinstimmende Daten bei dem Carana-, Colophonia- und Tacamahacaelemi, aber auch das von $\mathrm{Cr}$ emer isolierte Resen aus dem afrikanischen Elemi stimmt mit der von uns aufgestellten Formel gut überein. Denn die Formel $\mathrm{C}_{15} \mathrm{H}_{24} \mathrm{O}$ verlangt $81,81 \% \mathrm{C}$ und $10,90 \% \mathrm{H}$. Diese Formel entspricht aber der Zusammensetzung eines $\mathrm{Oxy}$-Sesquiterpens $=\left(\mathrm{C}_{5} \mathrm{H}_{8}\right)_{8} \mathrm{O}$ und bestätigen deshalb diese Resultate die Annahme Tschirchs, welcher die Resene als Oxy-Sesquiterpene oder Oxy-Polyterpene auffaßt.

Aus allem geht hervor, daß die Elemigruppe nicht minder interessant ist wie die Gruppe der Koniferenharze, mit der sie z. B. das Vorkommen zahlreicher isomerer Harzsäuren gemein hat.

Mitteilungen aus dem pharmazeutisch-chemischen Institut der Universität Marburg.

Von Ernst Schmidt.

\title{
169. Ueber das Isokreatinin.
}

Von Dr. G. Korndörfer.

(Eingegangen den 10. V: 1904.)

In dem letzten Hefte des Archivs für experimentello Pharmakalogie und Pathologie (LI, 227) findet sich eine Arbeit von Herrn Prof. E. Poulsson in Christiania über das Isokreatinin, in welcher der exakte Nachweis geführt wird, daß diese von Thesen') aus dem Fischfleische isolierte, gelb gefarbte Base identisch ist mit dem geworhnlichen, farblosen Kreatinin. Im Anschluß an meine Untersuchungen uber Glykocymidin und Kreatinin ${ }^{2}$ ), sowie an die früher hier ausgeführten Arbeiten von Pommerehne und Toppelias ${ }^{8}$ ) über

1) Ztschr. f. physiol. Chem. 24, 1.

2) Inaug.-Dissert. Marburg 1903.

B) Diese Ztschr. 1896, 380. 
Kreatinine verschiedenen Ursprungs, hatte auch ich mich mit dem gleichen Gegenstande beschäftigt und war dabei zu dem nämlichen Resultate gelangt, wie Herr Poulsson. Da sich meine Versuche znm Teil in anderer Richtung bewegten, als die von Herrn Poulsson, so mőchte ich, zur Ergänzung und Bestätigung der Angaben dieses Forschers, tiber die von mir gemachten Beobachtungen im nachstehenden kurz berichten.

Herr Poulsson hat die Identität dieser beiden Kreatinine zunächst dadurch bewiesen, daß es ihm gelang das Isokreatinin, welches auch nach beliebig vielen Umkrystallisierungen die intensiv gelbe Farbe unverändert beibehielt, durch Behandeln mit Tierkohle und durch Ueberführang in das Sulfat vollständig weiß zu erhalten. Weiter wurde die Löslichkeit beider Kreatinine, das Verhalten derselben gegen Permanganat, gegen Aetzbaryt und gegen Pikrinsäure verglichen, und hierbei vollständige Uebereinstimmong konstatiert. Mir ist die Entfärbung des Isokreatinins durch Ueberführung desselben in das Platindoppelsalz, bez. in das Hydrochlorid gelungen. Beide Verbindungen stimmten mit denen des gewöhnlichen Kreatinins in Form und Znsammensetzung vollkommen überein. Das Gleiche war der Fall bei dem Kreatinin und dem aus Isokreatininhydrochlorid regenerierten, farblosen "Isokreatinin". Auch in den Goldsalzen und den Pikraten beider Kreatinine, in den Löslichkeitsverhältnissen, in der Refraktion, in dem Reduktionsvermögen, in dem Verhalten gegen Kaliumpermanganat, sowie in den gesamten Reaktionen, ließ sich keine Verschiedenheit konstatieren.

Als Hauptunterschiede, welche zwischen Isokreatinin und Harnkreatinin obwalten, gibt $\mathrm{T}$ hesen folgende an:

1. Die Farbe: Isokreatinin stets gelb, Harnkreatinin und alle anderen bekannten Kreatinine farblos.

2. Die Löslichkeitsverhältnisse in Alkohol und Wasser: Isokreatinin löst sich in Wasser im Verhältnis $1: 4,2-4,4$, in Alkohol (kalt) 1:316. Harnkreatinin erfordert zur Lösung nach Pom merehne und Toppelius ${ }^{1}$ ) 10,6-11,5 T. Wasser und 625 T. Alkohol.

3. Das Pikrat: Isokreatininpikrat ist leicht loslich, daher wird Isokreatinin darch Pikrinsäure nicht gefallt. Kreatininpikrat ist tast unlosslich.

4. Die Verbindungen des Isokreatinins mit Schwermetallsalzen; namentlich soll die Chlorzinkverbindung verhältnismäßig leicht löslich sein.

1) Arch. d. Pharm. 1896, 390. 
5. Das Verhalten des Isokreatinins bei der Oxydation mit Permanganat: Es soll bei der Oxydation des Isokreatinins reichlich Ammoniak abgespalten und kein Methylguanidin als Spaltungsprodukt erhalten werden.

Mit dem Harnkreatinin gemeinsam hat das Isokreatinin die Farben. reaktionen. Ebenso soll dasselbe durch Einwirkung von Kalkmilch in ein Kreatin übergehen, welches farbios und wahrscheinlich mit Kreatin aus Harnkreatinin identisch ist.

Nachdem Pommerehne und Toppelius nachgewiesen haben (1. c.), daß die Kreatinine verschiedenen Ursprungs, synthetisches Kreatinin, Harnkreatinin und Kreatinin aus Kreatin des Muskelfleisches, identisch sind, so lag es nahe, das Isokreatinin Thesen's nochmals mit dem Harnkreatinin einer vergleichenden Untersuchung zu unterziehen, umsomehr als das Isokreatinin wesentliche Eigenschaften mit dem Kreatinin teilt. Den Unterschied in Farbe in Löslichkeitsverhältnissen konnten bedingt sein durch verhältnismäßig geringe Verunreinigungen, welche als solche die Analysen Thesen's nicht beeinflußten. Vor allem war es auffallend. daß ein dem Harn. kreatinin isomeres Kreatinin durch Hydrolyse dasselbe Kreatin liefern sollte, wie jenes.

Als Ausgangsmaterial zur Darstellung des Isokreatinins dienten mir $25 \mathrm{~kg}$ frisches Dorschfleisch (der Fisch ohne Kopf und Eingeweide). Ich hielt mich hierbei im wesentlichen au die Vorschrift Thesen's. Der Einfachheit halber babe ich jedoch das Muskelfleisch nicht abpräpariert, vielmehr kochte ich die Fische direkt mit Wasser aus. Dieses Verfahren hatte allerdings den Nachteil, daß sehr viel leimartige Substanzen mit extrahiert wurden, welche die Isolierung des fraglichen Kreatinins etwas erschwerten.

Der wässerige Auszug der Fische wurde zunächst auf freiem Feuer, später im Wasserbade auf ungefähr 8 Liter eingedampft und der Verdampfungsrückstand alsdann mit dem gleichen Volum starken Alkohols vermischt. Es entstand hierdurch eine sehr starke, voluminöse Fällung, welche durch Kolieren entfernt wurde. Die durch Abdestillieren von dem Alkohol befreite Kolatur wurde auf dem Dampfbade zu einem dicken Extrakt eingedampft, und dieses hierauf dreimal mit Alkohol ausgekocht. Die alkoholischen Auszüge trübten sich beim Erkalten und schieden eine schmierige, branne Masse ab, welche nicht weiter berücksichtigt wurde. Außerdem krystallisierte aus der alkoholischen Flüssigkeit Chlornatrium aus. Nach Abdestillation des größten Teiles des Alkohols wurde der Destillationsrückstand abermals bis zur Sirupkonsistenz eingedampft. Beim Erkalten erstarrte die Masse zu einem Krystallbrei. Da jedoch ein Absaugen der Mutter- 
lange nicht möglich war, wurde derselbe abermals mit Alkohol ausgekocht. Der erhaltene Auszug lieferte beim freiwilligen Verdunsten zunåchst keine Krystalle, erst nach dem Einengen zur dünnen Sirupkonsistenz erstarrte er nach dem Erkalten von neuem zu einem gelben Krystallbrei. Die von den Krystallehen abgesangte Mutterlauge (M) wurde, wie weiter nnten angegeben, verarbeitet, die Kryställchen selbst aber aus heißem Alkohol umkrystallisiert, wobei Chlornatrium ungelöst zurückblieb. Die durch das Umkrystallisieren erhaltene erste Krystallausscheidung bestand aus feinen, gelben, schwach alkalisch reagierenden Nadelchen. Aus der Mutterlauge von diesen Krystallen schieden sich zunåchst farblose Blättchen aus, welche abgesaugt wurden ${ }^{1}$ ). Nach dem Einimpfen einer kleinen Menge der gelben Nädelchen lieferte die Mutterlauge jedoch noch eine weitere Ausscheidung derselben Verbindung, so daB im ganzen etwa $3-4 \mathrm{~g}$ davon erhalten wurden.

Obige Mntterlauge (M) zeigte zunächst, selbst nach längerem Stehen, keine Neigung zur Krystallisation. Dieselbe wurde daher mit Salzsäure angesäuert und dann von neuem der freiwilligen Verdunstung iberiassen, wodurch sie allmählich zu einem Krystallbrei erstarrte. Letzterer wurde hierauf mit Alkohol vermischt, das Ungelöste (in wesentlichen Chlornatrium) abgesogen und die Lossung sodann von neuem der Verdunstung uberlassen, obne daß jedoch hierdurch eine Krystallisation erzielt wurde. Letztere schien durch eine schmierige, fettartige Substanz, die sich an der Oberfläche der Lösung abschied, verhindert zu werden. Um diese Substanz zu entfernen, wurde die Lösung mit Wasser verdünnt, hierauf mit Aether ausgeschättelt and dann von neuem zur Krystallisation eingedampft. Zur weiteren Reinigung wurde der krystallinische Rückstand nochmals in heißem Alkohol gelöst und so eine Flikssigkeit erhalten, welche direkt mehrere Krystallisationen von fast farblosen Nadeln lieferte. Letztere konnten durch Unkrystallisieren aus verdünntem Alkohol, unter Anwendung ron etwas Tierkohle, vollständig entfărbt werden. Diese Krystalle erwiesen sich als Kreatininhydrochlorid. Die Mutterlangen lieferten weitere Ausscheidungen, welche dem Kreatininhydrochlorid sehr ähnlich waren, jedoch noch eine blaßgelbe Färbung zeigten.

Der Chlorgehalt dieser gelblichen Krystalle war etwas niedriger als der des Kreatininhydrochlorids, trotzdem bestand auch dieses Produkt im wesentlichen nur aus normalem Kreatininhydrochlorid,

1) Die farblosen Blättchen erwiesen sich nach dem Umkrystallisieren aus Alkohol darch ihren Schmp. (1470) und ihr Verhalten gegen Chloroform und Schwefelsäure einerseits und gegen Essigsăureanhydrid and Schwefelsăure andererseits, als normales Cholesterin. 
wie die Ueberfuhrung desselben in das Golddoppelsalz und in freies Kreatinin lehrte.

Golddoppelsalz. Etwa $0,5 \mathrm{~g}$ dieser gelblichen Krystalle wurden in Wasser gelöst und diese Lösung mit Goldchlorid versetzt, wodurch sich sofort das charakteristische Goldsalz des Kreatinins in Blättchen ausscbied.

0,2496 desselben verloren bei $100^{\circ}$ nichts an Gewicht und gaben $0,1080 \mathrm{~g} \mathrm{Au}=43,27 \%$. Berechnet 43,52\% Au.

Zur Bestimmung des Schmelzpunktes wurde das erhaltene Chloroaurat und frisch bereitetes Kreatiningoldchlorid (hergestellt aus salzsaurem Harnkreatinin) in zwei gleich weiten Kapillarröbrchen erhitzt. Beide Proben schmolzen nnter Zersetzung bei $182-185^{\circ}$.

Der Rest der gelblichen Krystalle wurde durch Kochen mit fenchtem Bleihydroxyd von dem Chlor befreit, der Bleiniederschlag abgesaugt und die Lösung durch Schwefelwasserstoff vollständig von Blei betreit. Aus derselben krystallisierte nach dem Eindampfen das Kreatinin in farblosen, quadratischen Tafeln aus, welche frei von Krystallwasser waren; dieselben zeigten die typische Krystallform des gewöhnlichen Kreatinins.

Um das so aus Dorschfleisch erhaltene Kreatinin noch weiter mit dem Harnkreatinin zu identifizieren, habe ich noch folgende Untersuchungen vorgenommen.

\section{Bestimmung des Brechungswinkels in $2 \%$ iger Lösung.}

Derselbe wurde im Pulfrich'schen Refraktometer bei $13,6^{\circ} \mathrm{zu}$ $69^{\circ} 45^{\prime}$ gefunden.

Eine $2 \%$ ige Lösung von Harnkreatinin ergab bei derselben Temperatur einen Brechungswinkel von $69^{\circ} 8$.

\section{Bestimmung des Reduktionsvermōgens von je 0,05 g Kreatinin gegen $60 \mathrm{ccm}$ Fohling'sche Lösung.}

(Kochdauer 5 Minuten.)

Dorschkreatinin:
a) $0,05 \mathrm{~g}=0,0620 \mathrm{~g} \mathrm{Ca}$
b) $0,05 \mathrm{~g}=0,0656 \mathrm{~g} \mathrm{Ca}$
a) $0,05 \mathrm{~g}=0,0628 \mathrm{~g}$
b) $0,05 \mathrm{~g}=0,0644 \mathrm{~g}$.

Harnkreatinin:

\section{Bestimmung der Löslichkeit in absolutem Alkohol.}

$0,2 \mathrm{~g}$ Dorschkreatinin wurden mit $50 \mathrm{ccm}$ abs. Alkohol 2 Stunden lang gekocht, die Lösung darauf 24 Stunden lang unter hăufigem Umrühren stehen gelassen und schließlich von dem Ungelösten abfiltriert. 
$28,7550 \mathrm{~g}$ der Losung hinterließen $0.046 \mathrm{~g}$ Kreatinin. Loslich. keit $1: 624$.

Harnkreatinin löst sich in abs. Alkohol $1: 625$.

\section{Verhalten gegen Pikrinsäure.}

Versetzt man eine konzentrierte Lösung von Dorschkreatinin mit einer konzentrierten wässerigen Lösung von Pikrinsäure, so scheidet sich sofort ein Pikrat in schønen gelben Nädelchen aus. Das erhaltene Pikrat schmolz unter Zersetzung bei 215-217 . Das Pikrat aus Harnkreatinin verhält sich genau ebenso.

\section{Verhalten gegen eine alkoholische Chlorzinklösung.}

Versetzt man die wăsserige Lősung des Dorschkreatinins mit einer konzentrierten alkobolischen Chlorzinklösung, so entsteht fast sofort ein Niederschlag von Chlorzinkdoppelsalz. Unter dem Mikioskop zeigt dasselbe die sehr charakteristische Form des Chlorzinkdoppelsalzes des Harnkreatinins.

\section{Die Farbenreaktionen des Dorschkreatinins.}

Das Kreatinin aus Dorschfleisch zeigte die Weyl-Salkow ski'sche und die Jaffé'sche Farbenreaktion mit gleicher Empfindlichkeit wie das Harnkreatinin. Auch in dem sonstigen Verhalten gegen andere Agentien konnte bei beiden Kreatininen keine Verschiedenheit beobachtet werden.

\section{Verhalten des Dorschkreatinins bei Oxydation mittelst Kaliumpermanganat.}

$0,8 \mathrm{~g}$ Dorschkreatiniı wurden in $40 \mathrm{ccm}$ Wasser gelöst, die Lösung mit Natronlauge schwach alkalisch gemacht, auf $50-60^{\circ}$ erwärmt and nach Angabe von $\mathrm{Neubauer}$ ) solange tropfenweise mit einer $5 \%$ igen Kaliumpermanganatlosung versetzt, als dieses noch reduziert wurde. Es schied sich hierbei sofort Mangansuperoxyhydrat ans; eine erhebliche Entwickelung von Ammoniak fand jedoch nicht statt. Die entweichenden Dåmpfe färbten zwar rotes Lackınuspapier blau, aler ein Geruch nach Ammoniak trat direkt nicht anf. 0,8 $\mathrm{g}$ Harnkreatinin, in gleicher Weise behandelt, verhielten sich ganz ebenso.

Das Filtrat von dem ausgeschiedenen Mangansuperoxydhydrat wurde von Herrn A. Kircher einer weiteren Prüfung nach den Angaben von Neubauer unterzogen. Dorschkreatinin und Harnkreatinin

1) Ann. d. Chem. 119, 46. 
lieferten hierbei übereinstimmend lange, prismatische Krystalle von oxalsaurem Methylguanidin.

Zum Nachweise, daß die gelbe Farbe des sogen. Isokreatinins nur durch Verunreinigungen bedingt war, wurden ca. $3 \mathrm{~g}$ der gelben Nädelchen in das Platindoppelsalz durch Lösen in verdünnter Salzsäure und. Zufügen von Platinchloridchlorwasserstoffisăure übergeführt. Letzteres krystallisierte beim freiwilligen Verdunsten in roten, warzenförmigen Krystallaggregaten aus.

$0,5 \mathrm{~g}$ des erhaltenen Platinsalzes wurden zur Analyse aus salzsäurehaltigem Wasser umkrystallisiert. Ich erhielt hierbei das Salz wieder in kleinen Warzen.

$0,2439 \mathrm{~g}$ verloren bei $100^{\circ} 0,0130 \mathrm{~g}$ an Gewicht und lieferten $0,0711 \mathrm{~g} \mathrm{Pt}$.

Gefunden:

$\mathrm{H}_{2} \mathrm{O} \quad 5,33 \%$

Pt 30,79 ,

\section{Berechnet für}

$$
\begin{array}{cc}
2 \mathrm{Mol} . \mathrm{H}_{2} \mathrm{O}: & \left(\mathrm{C}_{4} \mathrm{H}_{7} \mathrm{~N}_{8} \mathrm{O}\right)^{2} \cdot \mathrm{H}_{2} \mathrm{PtCl}_{6}: \\
5,36 \% & - \\
- & 30,64 \% .
\end{array}
$$

Zur Abscheidnng des freien Kreatinins aus dem Platindoppelsalz wurden $5 \mathrm{~g}$ desselben mit $2 \mathrm{~g}$ Chlorkalium und ca. $50 \mathrm{ccm}$ Wasser anf dem Wasserbade zar Trockne eingedampft und der Trockenrückstand mit absolutem Alkohol wiederholt ausgekocht. Die alkoholischen Auszüge lieferten nach dem Eindampfen ein vollkommen weißes, salzsaures Salz. Dasselbe wurde nit Bleihydroxyd, wie vorher angegeben ist, zerlegt; die freie Base, welche so erhalten wurde, war ungefărbt und glich in der Krystallform, und in den Löslichkeitsverhältnissen durchaus dem Harnkreatinin und dem Kreatinin, welches früher ans dem Hydrochlorid isoliert worden war (siehe S. 377). Mit Goldchloridchlorwasserstoff lieferte es ein Chloroaurat, das in Blättchen krystallisierte und zwischen 180 und $185^{\circ}$ schmolz. 